\title{
Beamforming with Hybrid Channel Information in Spatially Correlated MISO Channels
}

\author{
Jae-Yun Ko and Yong-Hwan Lee \\ School of Electrical Engineering and INMC, Seoul National University \\ Kwanak P. O. Box 34, Seoul, 151-600 Korea
}

\begin{abstract}
In this paper, we propose a new beamforming scheme that utilizes both long-term and instantaneous channel state information (CSI). First, the dimension of the channel is effectively reduced by considering dominant eigen-dimensions of the channel covariance matrix. Then, the beam weight is generated using the instantaneous CSI of the effectively dimension-reduced channel. The reduction of the channel dimension is determined to maximize the signal-to-noise ratio (SNR) in an average sense considering the amount of feedback signaling burden and spatial correlation of the channel. It is analytically shown and verified by computer simulation that the proposed scheme outperforms conventional beamforming schemes when the same amount of feedback signaling burden is utilized.
\end{abstract}

\section{INTRODUCTION}

The performance of wireless systems can significantly be improved by using multiple antennas. For example, when channel state information (CSI) is fully available to the transmitter, the use of a coherent beamforming technique (also called maximum ratio transmission) can significantly increase the performance of wireless systems with its processing simplicity [1]. The transmitter can estimate the CSI using the link reciprocity in time-division duplex (TDD) systems, or it can get the CSI from the remote receiver through a feedback channel [2]. The feedback signaling burden for the CSI can be reduced by using a codebook technique [3, 4], where the weight vector is reported in a form of the index of a codebook comprising a finite set of vectors. However, it still increases feedback signaling burden approximately in linear proportion to the number of transmit antennas [5]. Moreover, the CSI should be updated periodically due to the time-varying channel characteristics, requiring a large feedback overhead. The feedback signaling burden can be reduced by utilizing transmit antennas in good channel condition [6]. However, it needs to send additional information on the selection of antennas.

Experimental results indicate that multi-antenna channels are often correlated in real environments [7, 8]. This property enables the generation of beamforming weight vectors based on the long-term CSI [9, 10], which is called eigenbeamforming in what follows. The weight vector is determined by the eigenvector corresponding to the maximum eigenvalue of the channel covariance matrix, enabling to maximally deliver the signal power to the receiver in an average sense. Although the optimum scheme can provide larger capacity with the use of transmit power allocation [11], the eigen- beamforming scheme is effective in highly correlated environments, while requiring reduced complexity. The longterm CSI is often available to the transmitter with reasonable accuracy without significant increase of the feedback signaling burden [12]. As the correlation between the transmit antennas decreases, however, the eigen-beamforming scheme can suffer from capacity loss compared to coherent beamforming scheme that utilizes instantaneous CSI.

The use of only instantaneous CSI or long-term CSI may not be efficient in real systems. In this paper, we consider the use of both CSI (or so-called hybrid CSI) for the generation of the beamforming weight. First, we reduce the effective dimension of the channel by considering only the eigen-dimensions corresponding to dominant eigenvalues of the channel covariance matrix. Then, we coherently generate the beamforming weight corresponding to the effective channel. The dimension of the effective channel can be determined to maximize the average SNR of the received signal according to the operating environment (e.g., the amount of the feedback signaling burden and the spatial correlation of the channel). Although the beamforming gain can somewhat be reduced by decreasing the dimension of the channel, the overall performance can be increased by reducing the quantization error of the beamforming vector.

The rest of this paper is organized as follows. The system model in consideration is described in Section II and the proposed scheme is described with the dimension reduction of the channel based on the long-term CSI in Section III. The performance of the proposed scheme is verified by computer simulation in Section IV and conclusions are finally summarized in Section V.

\section{SYSTEM MODEL}

Consider a multiple-input and single-output (MISO) system with $M$ transmit antennas and a single receive antenna. The received signal can be represented as

$$
y=\mathbf{h}^{*} \mathbf{x}+n
$$

where $\mathbf{h}$ denotes the $M$-dimensional Rayleigh fading channel vector whose elements are zero mean complex Gaussian random variables with unit variance but not independent, $\mathbf{x}$ is the $M$-dimensional transmit signal vector, $n$ is additive white 
Gaussian noise (AWGN) with variance $N_{o}$, and the superscript * denotes the conjugate transpose.

Assuming that the data symbol $s$ is transmitted using an $M$-dimensional beamforming vector $\mathbf{w}$, the transmit signal $\mathbf{x}$ can be represented as

$$
\mathbf{x}=\mathbf{w} s
$$

where $E\left\{|s|^{2}\right\}=P$ and $\|\mathbf{w}\|_{F}=1$, resulting in an average transmit power of $P$. Here $E\{\cdot\}$ and $\|\cdot\|_{F}$ denote the expectation operation and the Frobenius norm, respectively. The channel covariance matrix defined by

$$
\mathbf{R} \triangleq E\left\{\mathbf{h h}^{*}\right\}
$$

is a positive definite Hermitian matrix and thus can be decomposed as

$$
\mathbf{R}=\mathbf{Q} \boldsymbol{\Sigma}^{2} \mathbf{Q}^{*}
$$

where $\mathbf{Q}=\left[\mathbf{q}_{1} \cdots \mathbf{q}_{M}\right]$ is an $(M \times M)$-dimensional unitary matrix whose columns $\left\{\mathbf{q}_{i}\right\}$ are eigenvectors of $\mathbf{R}$ and $\boldsymbol{\Sigma}$ is an $M$-dimensional diagonal matrix whose diagonal elements $\sigma_{1}, \ldots, \sigma_{M}$ are ordered non-negative eigenvalues of $\mathbf{R}^{1 / 2}$ (i.e., $\left.\sigma_{1} \geq \cdots \geq \sigma_{M} \geq 0\right)$. Using $\operatorname{Tr}[\mathbf{R}]=\operatorname{Tr}\left[\boldsymbol{\Sigma}^{2}\right]=\sum_{i=1}^{M} \sigma_{i}^{2}$ from (4) and $\operatorname{Tr}[\mathbf{R}]=E\left\{\|\mathbf{h}\|_{F}^{2}\right\}=M$ from (3), it can be shown that $\sum_{i=1}^{M} \sigma_{i}^{2}=M$.

The channel $\mathbf{h}$ can be expressed as [10]

$$
\mathbf{h}=\mathbf{R}^{1 / 2} \mathbf{h}_{w}=\mathbf{Q} \mathbf{\Sigma} \mathbf{Q}^{*} \mathbf{h}_{w}
$$

where $\mathbf{h}_{w}=\left[h_{w 1} \cdots h_{w M}\right]^{T}$ is an $M$-dimensional vector whose entries are independent and identically distributed (i.i.d.) zero mean complex Gaussian random variables with unit variance and the superscript $T$ denotes transpose of a vector. Since $\mathbf{Q}^{*} \mathbf{h}_{w}$ and $\mathbf{h}_{w}$ are identically distributed, (5) can be rewritten as

$$
\mathbf{h}=\mathbf{Q} \mathbf{\Sigma} \mathbf{h}_{w} .
$$

\section{BEAMFORMING WITH HYBRID CSI}

In this section, we present a new beamforming scheme that utilizes both the instantaneous and the long-term CSI. We first assume no quantization for the beamforming vector, and then consider the effect of quantization.

\section{A. Proposed Beamforming without Quantization}

The proposed beamforming considers the use of an eigenspace associated with eigenvectors corresponding to dominant eigenvalues of the channel covariance matrix. The eigenspace corresponding to the $L$ largest eigenvalues can be chosen by multiplying an $(M \times L)$-dimensional matrix $\mathbf{Q}_{1: L}$ whose columns are the first $L$ columns of $\mathbf{Q}$. Note that $\mathbf{Q}_{1: L}$ maximizes the average channel gain, defined by
$E\left\{\left\|\mathbf{h}^{*} \mathbf{Q}_{1: L}\right\|^{2}\right\}$, among all $(M \times L)$-dimensional matrices with orthonormal columns [13]. Since $\mathbf{Q}_{1: L}$ is a function of $\mathbf{R}$, the feedback signaling overhead for the reporting of $\mathbf{Q}_{1: L}$ may not be significant (e.g., it can be assumed that the longterm CSI is unchanged during about $1000 \mathrm{~ms}$ even when the user is in a mobility of $1000 \mathrm{~km} / \mathrm{h}[12])$.

Letting $\left.\mathbf{h}_{L} \triangleq \mathbf{Q}_{1: L}^{*} \mathbf{h}\right)$ be an effective channel generated by $\mathbf{Q}_{1: L}$, the received signal can be represented as

$$
y=\mathbf{h}_{L}^{*} \mathbf{x}_{L}+n
$$

where $\mathbf{x}_{L}=\mathbf{w}_{L} S$ is the $L$-dimensional signal vector to be transmitted through $\mathbf{h}_{L}$ and $\mathbf{w}_{L}$ is the beamforming weight associated with $\mathbf{h}_{L}$. For a given $\mathbf{h}_{L}$, the optimum weight $\mathbf{w}_{L}$ can be determined by

$$
\mathbf{w}_{L}=\frac{\mathbf{h}_{L}}{\left\|\mathbf{h}_{L}\right\|_{F}} .
$$

Although the overall beamforming weight $\mathbf{w}=\mathbf{Q}_{1: L} \mathbf{w}_{L}$ is an $M$-dimensional vector, the proposed scheme needs to update only $L$-dimensional beamforming vector $\mathbf{w}_{L}$, implying the reduction of the feedback signaling overhead approximately by a factor of $L / M$ compared to the coherent beamforming whose beam weight is an $M$-dimensional vector [1]. The antenna selection scheme also considers partial dimensions of the channel for the beamforming [6]. However, it is different from the proposed beamforming scheme that determines the dimension of the effective channel based on the long-term CSI without the use of instantaneous CSI.

The SNR of the received signal by the proposed beamforming can be represented as

$$
\begin{aligned}
\gamma_{\text {hyb }}^{L} & =\bar{\gamma}\left|\mathbf{h}_{L}^{*} \frac{\mathbf{h}_{L}}{\left\|\mathbf{h}_{L}\right\|_{F}}\right|^{2} \\
& =\bar{\gamma}\left\|\mathbf{h}_{L}\right\|_{F}^{2}=\bar{\gamma}\left\|\mathbf{h}^{*} \mathbf{Q}_{1: L}\right\|_{F}^{2}
\end{aligned}
$$

Using $\mathbf{h}=\mathbf{Q} \boldsymbol{\Sigma} \mathbf{h}_{w}$ from (6), it can be shown that

$$
\mathbf{h}^{*} \mathbf{Q}_{1: L}=\mathbf{h}_{w}^{*} \boldsymbol{\Sigma} \mathbf{Q}^{*} \mathbf{Q}_{1: L}=\mathbf{h}_{w}^{*} \boldsymbol{\Sigma}_{1: L}
$$

where $\boldsymbol{\Sigma}_{1: L}$ is an $(M \times L)$-dimensional rectangular matrix whose $L$ diagonal elements are the same as the first $L$ diagonal elements of $\boldsymbol{\Sigma}$ and whose off-diagonal elements are zero. Thus, (9) can be rewritten as

$$
\gamma_{\text {hyb }}^{L}=\bar{\gamma}\left\|\mathbf{h}_{w}^{*} \boldsymbol{\Sigma}_{1: L}\right\|_{F}^{2}=\bar{\gamma} \sum_{i=1}^{L}\left|h_{w i}\right|^{2} \sigma_{i}^{2} .
$$

Finally, the expected value of $\gamma_{\text {hyb }}^{L}$ is given by

$$
E\left\{\gamma_{\text {hyb }}^{L}\right\}=\bar{\gamma} \sum_{i=1}^{L} \sigma_{i}^{2}
$$


Note that the proposed beamforming scheme achieves a beamforming gain of $\sum_{i=1}^{L} \sigma_{i}^{2}$ which is the sum of the $L$ largest eigenvalues of the channel covariance matrix. This beamforming gain is larger than that of the eigen-beamforming $[1$,$] whose beamforming gain is \sigma_{1}^{2}$ and less than that of the coherent beamforming [9] whose beamforming gain is $\sum_{i=1}^{M} \sigma_{i}^{2}$. In fact, the coherent beamforming and the eigenbeamforming are special cases of the proposed beamforming, i.e., $L=M$ for the coherent beamfoming and $L=1$ for the eigen-beamfoming. By properly choosing $L$, the proposed scheme can optimally trade the performance against the feedback signaling burden. We consider this issue in the following subsection with the effect of quantization.

\section{B. Effect of quantization}

To reduce the feedback signaling burden, the beamforming vector is often reported in a form of the index of a predetermined codebook. Assuming the use of a codebook $C$ comprising $2^{B}$ unit-norm vectors $\left\{\mathbf{v}_{1}, \mathbf{v}_{2}, \ldots, \mathbf{v}_{2^{B}}\right\}$, the receiver determines a weight vector that maximizes the SNR among $2^{B}$ entries in the codebook and reports its $B$-bit index to the transmitter.

For given channel $\mathbf{h}_{L}$ and $C$, the beamforming vector is determined by

$$
\hat{\mathbf{w}}_{L}=\arg \max _{\mathbf{v} \in C}\left|\mathbf{h}_{L}^{*} \mathbf{v}\right| .
$$

The quantized beamforming vector $\hat{\mathbf{w}}_{L}$ can be represented as [5]

$$
\hat{\mathbf{w}}_{L}=\alpha_{L}(B) \frac{\mathbf{h}_{L}}{\left\|\mathbf{h}_{L}\right\|_{F}}+\sqrt{1-\left|\alpha_{L}(B)\right|^{2}} \mathbf{z}
$$

where $\mathbf{z}$ is an $L$-dimensional unit norm vector isotropically distributed over a space orthogonal to $\mathbf{h}_{L}$ and $\alpha_{L}(B)$ is a random variable related to the quantization noise. Since the codebook is usually generated in advance independent of the channel, $\alpha_{L}(B)$ can also be assumed to be independent of $\mathbf{h}_{L}$.

The SNR of the proposed beamforming with the use of the $B$-bit quantization can be represented as

$$
\gamma_{\text {hyb }}^{L}(B)=\bar{\gamma}\left|\mathbf{h}_{L}^{*} \hat{\mathbf{w}}_{L}\right|^{2}=\bar{\gamma}\left|\alpha_{L}(B)\right|^{2}\left\|\mathbf{h}_{L}\right\|_{F}^{2} .
$$

Since $E\left\{\left\|\mathbf{h}_{L}\right\|_{F}^{2}\right\}=\sum_{i=1}^{L} \sigma_{i}^{2}$, it can be seen that

$$
E\left\{\gamma_{\text {hyb }}^{L}(B)\right\}=\bar{\gamma} E\left\{\left|\alpha_{L}(B)\right|^{2}\left\|\mathbf{h}_{L}\right\|_{F}^{2}\right\}=\bar{\gamma} E\left\{\left|\alpha_{L}(B)\right|^{2}\right\} \sum_{i=1}^{L} \sigma_{i}^{2}
$$

Note that the SNR is reduced by a factor of $E\left\{\left|\alpha_{L}(B)\right|^{2}\right\}$ due to the quantization, where $0<E\left\{\left|\alpha_{L}(B)\right|^{2}\right\}<1$.
For further investigation of the quantization effect, consider the use of a random codebook whose entries are randomly chosen from an $L$-dimensional unit sphere. Then, it can be shown that [14]

$$
E\left\{\left|\alpha_{L}(B)\right|^{2}\right\}=1-2^{B} \beta\left(2^{B}, \frac{L}{L-1}\right)
$$

where $\beta_{\infty}(x, y)=\Gamma(x) \Gamma(y) / \Gamma(x+y)$ is the Beta function and $\Gamma(x)=\int_{0}^{\infty} t^{x-1} e^{-t} d t$ is the Gamma function. It can be shown from (17) that (16) can be rewritten as

$$
E\left\{\gamma_{\text {hyb }}^{L}(B)\right\}=\bar{\gamma}\left(1-2^{B} \beta\left(2^{B}, \frac{L}{L-1}\right)\right) \sum_{i=1}^{L} \sigma_{i}^{2} .
$$

Using an approximation [5]

$$
E\left\{\left|\alpha_{L}(B)\right|^{2}\right\} \approx 1-2^{-\frac{B}{L-1}},
$$

(18) can further be simplified to

$$
E\left\{\gamma_{\text {hyb }}^{L}(B)\right\} \approx \bar{\gamma}\left(1-2^{-\frac{B}{L-1}}\right) \sum_{i=1}^{L} \sigma_{i}^{2} .
$$

It can be seen that as $L$ increases, the term $\left(1-2^{-\frac{B}{L-1}}\right)$ due to the quantization decreases, but the beamforming gain $\sum_{i=1}^{L} \sigma_{i}^{2}$ increases. This implies the existence of an optimum $L$ that maximizes the average SNR of the received signal for a given $B$. For example, when $B$ is large, the quantization noise term is less sensitive to $L$ and thus the use of a large $L$ (i.e., larger beamforming gain) can provide better performance, and vice versa. The optimum $L$ also depends on the correlation of the channel. For example, when the channel has a large correlation, the channel gain is mostly concentrated on a small number of eigenvalues, say $l$ eigenvalues. In this case, the use of an $L \geq l$ does not noticeably improve the beamforming gain. This implies that in highly correlated environments, the use of a small $L$ may be desirable reducing the quantization noise.

For a given $B$, the optimum $L$ associated with the channel correlation can be determined as

$$
\begin{aligned}
L^{*} & =\arg \max _{L \in\{1, \ldots, M\}} E\left\{\gamma_{\text {hyb }}^{L}(B)\right\} \\
& =\arg \max _{L \in\{1, \ldots, M\}} E\left\{\left|\alpha_{L}(B)\right|^{2}\right\} \sum_{i=1}^{L} \sigma_{i}^{2} .
\end{aligned}
$$
In practice, the codebook specific parameter $E\left\{\left|\alpha_{L}(B)\right|^{2}\right\}$
can empirically be found from (13) and (14) by

$$
E\left\{\left|\alpha_{L}(B)\right|^{2}\right\}=E\left\{\left|\mathbf{u}^{*}\left(\arg \max _{\mathbf{v} \in C}\left|\mathbf{u}^{*} \mathbf{v}\right|\right)\right|^{2}\right\}
$$

where $\mathbf{u}$ is a random vector isotropically distributed over an $L$-dimensional unit sphere. 


\section{Performance Evaluation}

The performance of the proposed beamforming scheme is verified by computer simulation. We assume that the signal is transmitted over Rayleigh fading channel using a $(4 \times 1)$ MISO antenna scheme whose beam weight is generated using a random codebook and reported through an error-free feedback channel with zero-delay. We also assume that the transmit antennas are correlated with the channel covariance matrix given by [16]

$$
\mathbf{R}=\left[\begin{array}{cccc}
1 & \rho & \rho^{2} & \rho^{3} \\
\rho^{*} & 1 & \rho & \rho^{2} \\
\rho^{2^{*}} & \rho^{*} & 1 & \rho \\
\rho^{3^{*}} & \rho^{2^{*}} & \rho^{*} & 1
\end{array}\right] .
$$

The proposed scheme uses a reduced dimension given by (21).

Fig. 1 depicts the SNR of the received signal according to the quantization bit size when the average SNR is $10 \mathrm{~dB}$ and $\rho=0.6$, where the analytic results are from (18). It can be seen that although the use of $L=4$ provides the best performance with the use of a large quantization size (e.g., $B \geq 10$ ), the use of $L=2$ is optimum when the use of a quantization size is small (e.g., $2 \leq B \leq 5$ ). Fig. 2 depicts the SNR of the received signal associated with the channel correlation when the average SNR is $10 \mathrm{~dB}$ and $B=6$. It can be seen that the use of a large $L$ is desirable when the channel is less correlated, but the use of a small $L$ is desirable as the channel correlation increases. Adjusting $L$ according to the channel condition and feedback signaling constraint, the proposed scheme can provide a desirable performance.

We evaluate the performance of the proposed scheme in terms of the transmission capacity in suburban macro environment of tapped delay-line model in [15]. For comparison, we also evaluate the performance of three conventional schemes: coherent beamforming, eigenbeamforming and antenna selection schemes [6]. The antenna selection scheme first selects $n$ transmit antennas having the $n$ largest channel gain and then applies the coherent beamforming technique to the selected antennas. We assume that all the schemes except the eigen-beamforming which utilizes only the long-term CSI, use the same feedback bits for each beam weight. Note that the antenna selection scheme uses some bits for the selection of transmit antennas (e.g., three bits for the selection of two antennas and two bits for the selection of three antennas) and the rest of bits for the beamforming weight.

Fig. 3 depicts the transmission capacity when $B=4$ and four transmit antennas are equally separated by two and ten wavelengths, yielding a correlation between adjacent antennas of about 0.96 and 0.63 , respectively. The corresponding optimum value of $L$ is two and three, respectively. It can be seen from Fig. 3 (a) that when the channel is highly correlated, the proposed scheme noticeably outperforms the conventional beamforming schemes and that the eigen-beamforming outperforms the coherent beamforming mainly due to that the most of gain is concentrated on the first eigen-value of the channel covariance matrix. The antenna selection scheme provides the worst performance mainly due to the use of fewer bits for the beamforming weight and reduced selection diversity gain in highly correlated environments. It can be seen from Fig. 3 (b) that even when the channel is moderately correlated, the proposed scheme still noticeably outperforms the conventional beamforming schemes.

Fig. 4 depicts the capacity of the proposed scheme associated with the quantization bit size when four transmit antennas are equally separated by six wavelengths, yielding a channel correlation between adjacent antennas of about 0.74 . The optimum value of $L$ is $L^{*}=2$ for $3 \leq B \leq 6$ and $L^{*}=3$ for $7 \leq B \leq 10$. It can be seen that the proposed scheme provides a large capacity gain over the coherent beamforming scheme particularly when the quantization bit size is small, implying the effectiveness of the proposed schemes in practical applications where the feedback signaling burden should be minimized. It can also be seen that although the eigenbeamforming can outperform the coherent beamforming when a small number of quantization bits is used (e.g., $B=3$ ), its performance is still much smaller than that of the proposed beamforming scheme.

\section{CONCLusions}

We have proposed a new beamforming scheme that utilizes both the long-term and instantaneous CSI. Exploiting the spatial correlation of the channel, the proposed scheme utilizes less amount of the feedback signaling overhead without noticeable performance loss compared to the use of full CSI. The simulation results show that the proposed scheme can effectively be applied to real environments where the channel is non-negligibly correlated and the amount of feedback signaling overhead is strictly limited.

\section{REFERENCES}

[1] T. K. Y. Lo, "Maximum ratio transmission," IEEE Trans. Commun., vol. 47, pp. 1458-1461, Oct. 1999.

[2] K. K. Mukkavilli, A. Sabharwal, E. Erkip, and B. Aazhang, "On beamforming with finite rate feedback in multiple antenna systems," IEEE Trans. Inf. Theory, vol. 49, no. 10, pp. 2562-2579, Oct. 2003.

[3] D. Love, R. Heath, Jr., and T. Strohmer, "Grassmannian beamforming for multiple-input multiple-output wireless systems," IEEE Trans. Inf. Theory, vol. 49, pp. 2735-2747, Oct. 2003.

[4] D. Love and R. Heath, Jr., "Equal gain transmission in multiple-input multiple-output wireless systems," IEEE Trans. Commun., vol. 51, pp. 1102-1110, July 2003.

[5] N. Jindal, "MIMO broadcast channels with finite rate feedback," IEEE Inf. Theory, vol. 52, pp. 5045-5060, Nov. 2006.

[6] S. Sanayei and A. Nosratinia, "Antenna selection in MIMO systems," IEEE Commun. Mag., vol. 42, pp. 68-73, Oct. 2004.

[7] J. P. Kermoal, L. Schumacher, K. I. Pedersen, P. E. Mogensen, and F. Frederiksen, "A stochastic MIMO radio channel model with experimental validation", IEEE J. Sel. Areas Commun., vol. 20, no. 6, pp. 1211-1226, Aug. 2002. 
This full text paper was peer reviewed at the direction of IEEE Communications Society subject matter experts for publication in the ICC 2008 proceedings.

[8] W. Weichselberger, M. Herdin, H. Ozcelik, and E. Bonek, "A stochastic MIMO channel model with joint correlation of both link ends," IEEE Trans. Wireless Commun., vol. 5, no. 1, pp.90-100, Jan. 2006.

[9] A. Narula, M. J. Lopez, M. D. Trott, and G. W. Wornell, "Efficient use of side information in multiple-antenna data transmission over fading channels," IEEE J. Sel. Areas Commun., vol. 16, pp. 1423-1436, Oct. 1998.

[10] S. Jafar and A. Goldsmith, "Transmitter optimization and optimality of beamforming for multiple antenna systems," IEEE Trans. Wireless Commun., vol. 3, no. 4, pp. 1165-1175, July 2004.

[11] A. M. Tulino, A. Lozano, and S. Verdu, "Capacity-achieving input covariance for single-user multi-antenna channels," IEEE Trans. Wireless commun., vol. 5, no. 3, pp. 662-671, Mar. 2006.

[12] M. Nicoli, O. Simeone, and U. Spagnolini, "Multislot estimation of fast varying communication channels," IEEE Trans. Signal Processing, vol. 5, pp. 1184-1195, May 2003.

[13] Strang, Linear Algebra and Its Applications, 3rd Ed., Brooks/Cole (Thomson Learning), 1988.

[14] C. K. Au-Yeung and D. J. Love, "On the performance of random vector quantization limited feedback beamforming in a MISO system," IEEE Trans. Wireless Commun., vol. 6, pp. 458-462, Feb. 2007.

[15] D. S. Baum, J. Hansen, and J. Salo, "An interim channel model for beyond-3G systems: extending the 3GPP spatial channel model (SCM)," in Proc. IEEE Veh. Technol. Conf., vol. 5, May 2005, pp. 3132-3136.

[16] X. Mestre, J. R. Fonollosa, and A. Pages-Zamora, "Capacity of MIMO channels: asymptotic evaluation under correlated fading," IEEE J. Sel. Areas Commun., vol. 21, no. 5, pp. 829-838, June 2003.

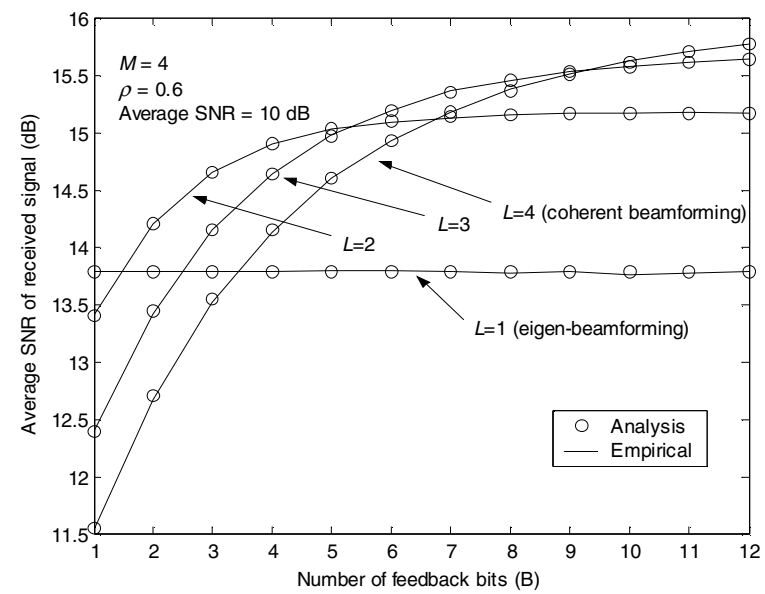

Fig. 1. Average SNR of the received signal due to the quantization.

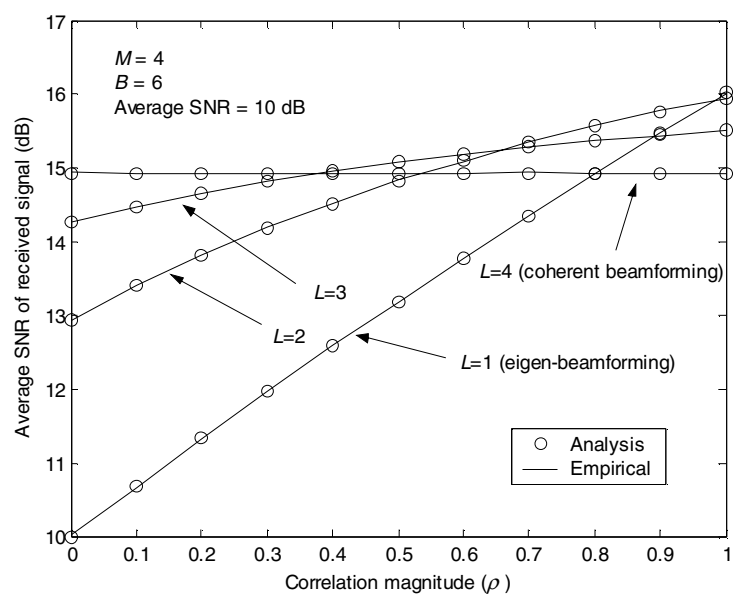

Fig. 2. Average SNR of the received signal associated with $\rho$.

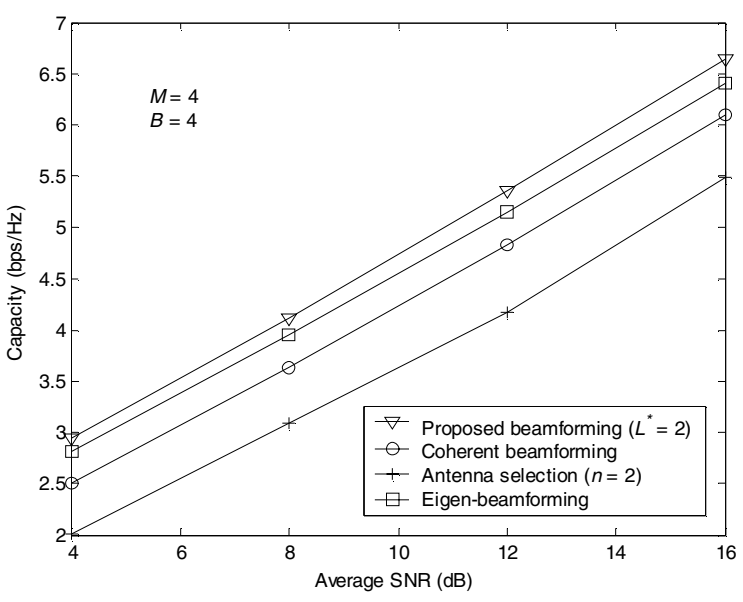

(a) When $\rho \approx 0.96$ and $L=2$.

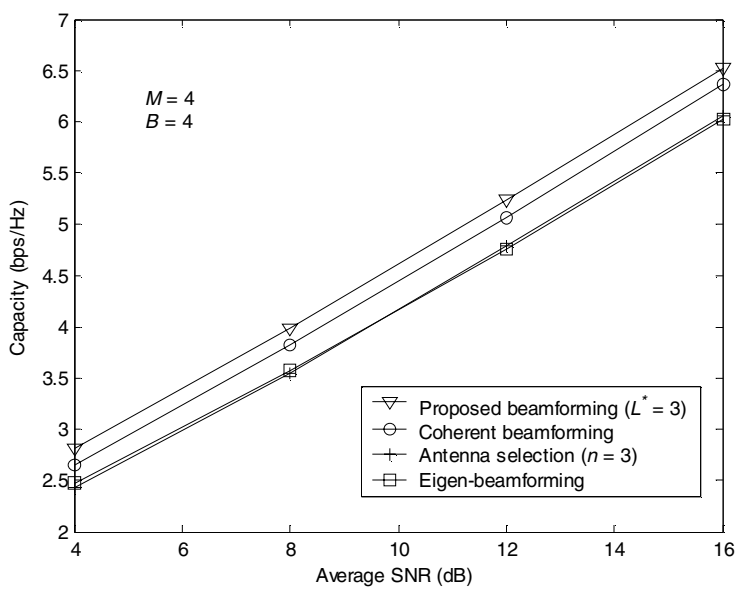

(b) When $\rho \approx 0.63$ and $L=3$.

Fig. 3. Transmission capacity of the proposed scheme.

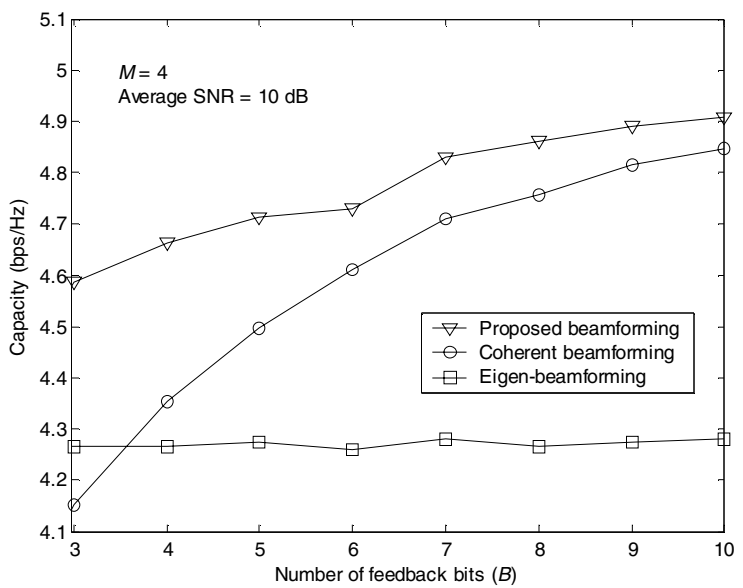

Fig. 4. Capacity associated with the quantization bit size when $\bar{\gamma}=10 \mathrm{~dB}$. 